Archive for

Organic Chemistry
Arkivoc 2018, part vii, 0-0

to be inserted by editorial office

\title{
Synthesis of arylpiperazine substituted bisindolylmethanes as possible pharmacologically active new compounds
}

\author{
Busra Arvas, Omer Tahir Gunkara, and Nuket Ocal ${ }^{*}$
}

Yildiz Technical University, Faculty of Science and Arts, Department of Chemistry, Davutpasa Campus, 34220,

Esenler, Istanbul, Turkey

Email: nocal@yildiz.edu.tr

Received 03-14-2018

Accepted 08-10-2018

Published on line 09-06-2018

\section{Abstract}

Nitrogen containing heterocyclic rings with a piperazine group are valuable target compounds in medicinal chemistry due to their diversified biological activities. In recent years, many organocatalytic/metal free synthetic pathways towards bisindolylmethanes have been reported. ${ }^{1-3}$ In the present work, we combined bisindolylmethanes with arylpiperazines using hydrophobic moiety (alkyl chain) to obtain heterocycles that can be evaluated in pharmacologically and may be more active than the already active bisindolylmethane alkaloids.

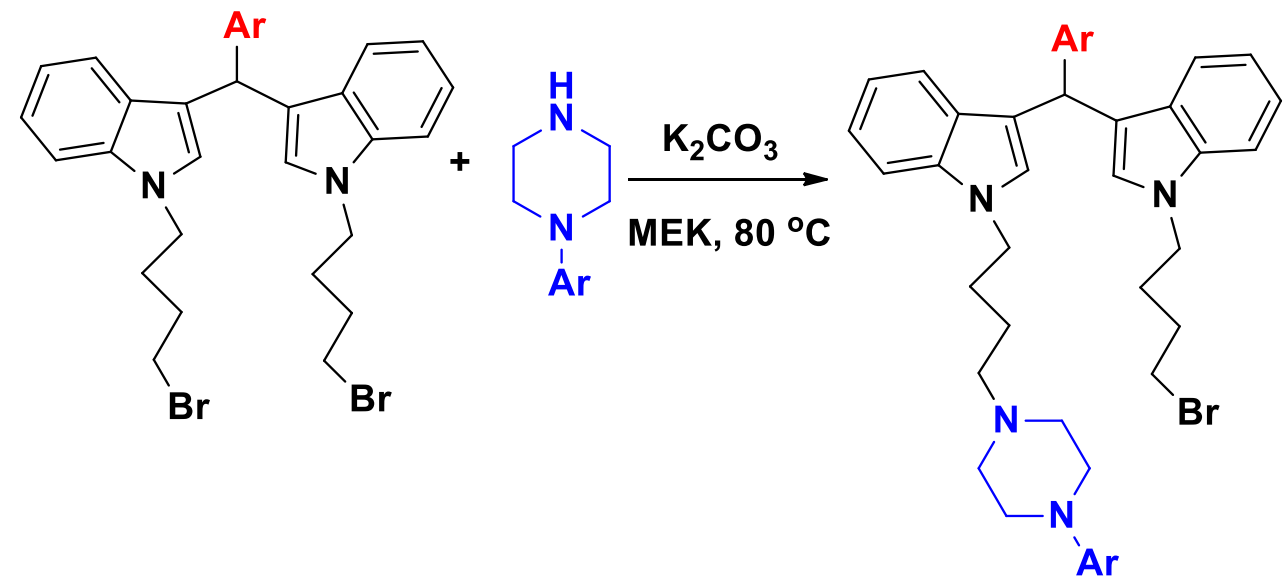

Keywords: Arylpiperazines, bisindoylmethanes, DBDMH, biologically active molecules 
Bisindolylmethanes constitute an important class of heterocyclic compounds containing an indole ring and exhibiting a variety of pharmacological activities such as antifungal, antihyperglycemic, anti-inflammatory, antibacterial, anti-cancer, antimicrobial and anti-leishmanial activities, including enzyme inhibition activity. ${ }^{4-7}$ Some of them are accountable for beneficial estrogen metabolism and induce apoptosis in cancer cells of human. ${ }^{8}$ They also exhibit inhibitory activity against bladder cancer ${ }^{9}$ and renal cell carcinoma growth ${ }^{10}$ and inhibit lung ${ }^{11}$ and colon cancers. ${ }^{12}$ Some bisindolylmethanes showed carbonic anhydrase II inhibitor. ${ }^{13}$ On the other hand, dietary nature indoles like 3,3'-diindolylmethane ${ }^{14}$ that are generated from cruciferous vegetables can both stimulate apoptosis and confer protection against DNA damage in human colon cell lines.

The most common method for the synthesis of BIMs involves consecutive nucleophilic addition of two molecules of indole (nucleophile) to an aldehyde (electrophile) as a one-pot reaction in the presence of a number of catalysts like Brønsted, Lewis acids such as $\mathrm{LiClO}_{4}, \operatorname{In}(\mathrm{OTf})_{3}, \mathrm{Dy}(\mathrm{OTf})_{3}, \mathrm{Sc}(\mathrm{OTf})_{3}, \mathrm{CAN}, \mathrm{ZrOCl}_{2}, \mathrm{InCl}_{3}$, heteropoly acids, ionic liquids, surfactants. ${ }^{15-17}$

In our previous work, several UV-active bis(indolyl)methanes were prepared in the presence of 1,3dibromo-5,5-dimethylhydantoin (DBDMH) ${ }^{18}$ and the UV-absorbing activity were investigated both in solution and polymer substrate. This study focuses on design and discovery of new bisindolylmethanes including arylpiperazine group. We think that these compounds could show various biological activities and even be employed in vivo studies owing to the fact that they carry two biologically important structural units, namely, the bisindolylmethane and a 1-arylpiperazinyl unit.

\section{Results and Discussion}

$1 \mathrm{H}$-Indole (1) react with 1,4-dibromobutane react to afford 1 -(4-bromobutyl)- $1 \mathrm{H}$-indole $(\mathbf{2})^{19}$ which under treatment with $p$-chlorobenzaldehyde, $p$-fluorobenzaldehyde, phenanthrene-9-carbaldehyde and 2naphtaldehyde, respectively, in the presence of 1,3-dibromo-5,5-dimethylhydantoin (DBDMH) leads to new compounds 3-6 in good yields (Schemes 1 and 2).

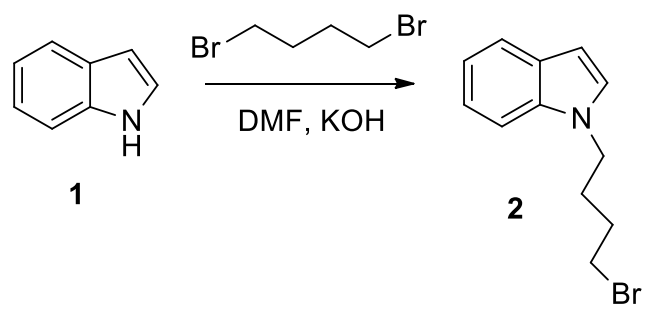

Scheme 1. Preparation of 2. 
2
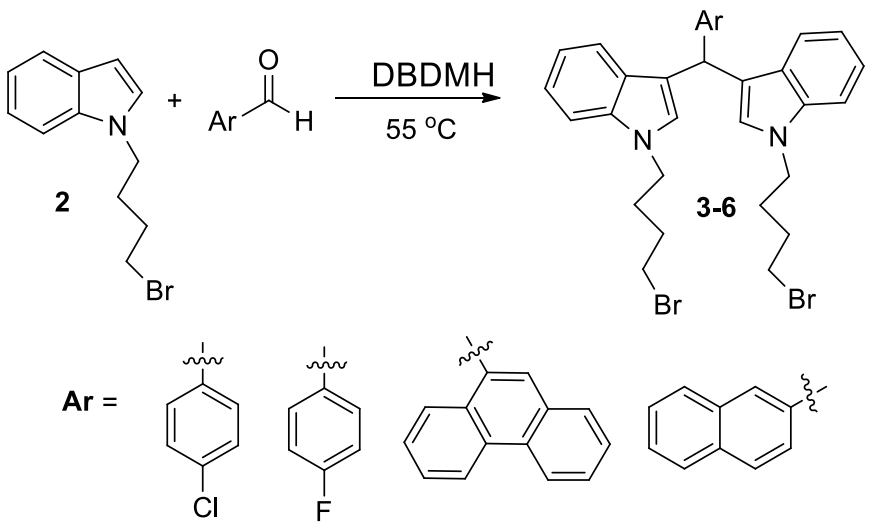

6

Scheme 2. Synthesis of 3-6.

Alternatively, reaction of $1-\mathrm{H}$-indole and aldehydes in the presence of gold chloride III $\left(\mathrm{AuCl}_{3}\right)$ as catalyst, in acetonitrile under nitrogen atmosphere resulted to the formation of compounds 3-6 in higher yields (Scheme 3).

2

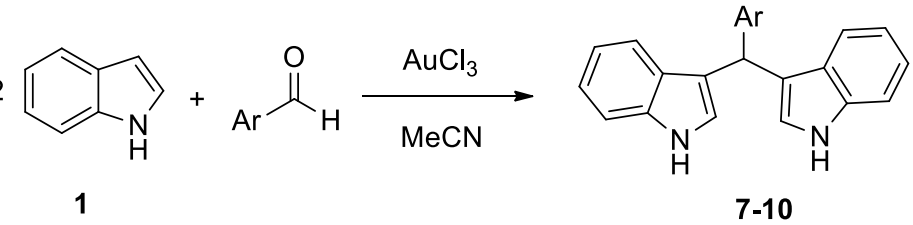<smiles>CC(C)(C)c1ccc(Cl)cc1</smiles><smiles>Nc1ccc(F)cc1</smiles><smiles>Nc1cc2ccccc2c2ccccc12</smiles>

9<smiles>Cc1ccc2ccccc2c1</smiles>

10

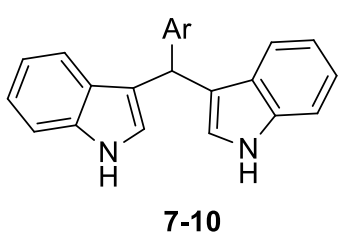<smiles>C[C@H](OC(C)(C)C)C(C)(C)C</smiles>

Scheme 3. Synthesis of 3-6.

Compounds 3, 5 and 6, respectively reacted with 2 moles of 1-(2,3-dichlorophenyl)piperazine hydrochloride with $\mathrm{K}_{2} \mathrm{CO}_{3}$ in MEK to give new derivatives 11-13 (Scheme 4). Spectroscopic studies showed that one piperazine ring was present in the product, and the reactions were repeated a few times. 


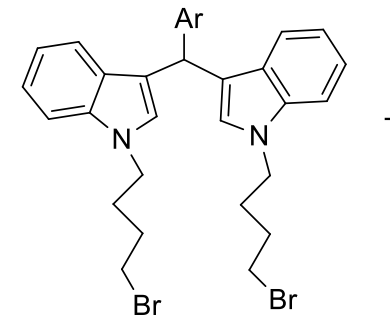

$3,5,6$<smiles>Clc1cccc(N2CCNCC2)c1Cl</smiles>

$\underset{\text { MEK, } 80^{\circ} \mathrm{C}}{\stackrel{\mathrm{K}_{2} \mathrm{CO}_{3}}{\longrightarrow}}$

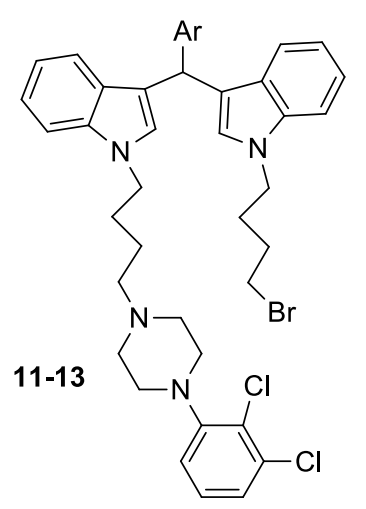

Scheme 4. Synthesis of 11-13.

Furthermore, compounds 3 and 4 were reacted with 2 moles of 2-(piperazin-1-yl)pyrimidine under same conditions to give compounds 14 and 15 (Scheme 5).
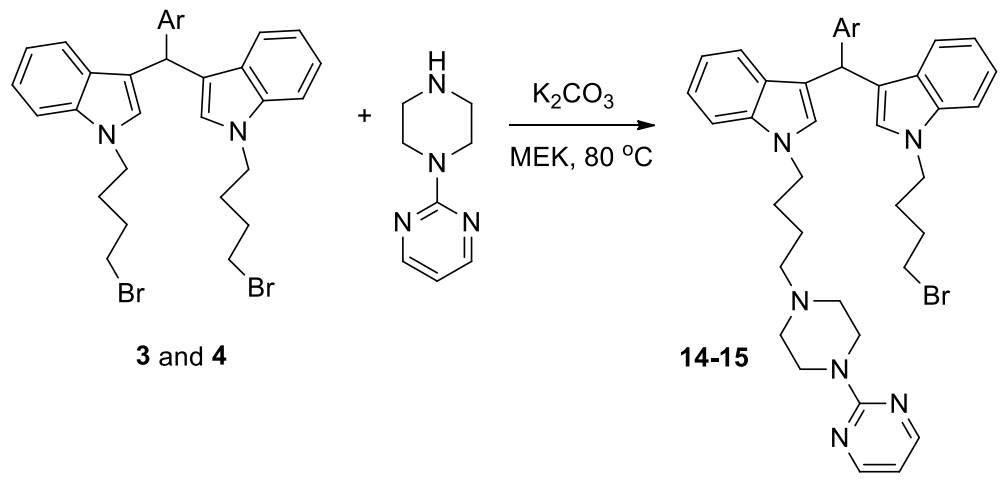

Scheme 5. Synthesis of 14-15.

The mono piperazinyl substitution pattern in compounds $\mathbf{1 4}$ and $\mathbf{1 5}$ was confirmed primarily by ${ }^{1} \mathrm{H} N M R$ spectroscopy. Equivalent protons adjacent to the nitrogens in the piperazine ring showed a doublet for two protons at 8.30-8.32 ppm in the spectra. The structures were also supported by HRMS results.

Initially, we also tried to attach the piperazine ring to compound $\mathbf{2}$ and then to obtain new bisindolylmethanes. According to literature procedure ${ }^{20}$ 1-(3-trifluoromethyl)piperazine has been used to obtain compound 16 (Scheme 6). Subsequently, reaction of two moles of compound 16 with benzaldehyde led to the formation of compound $\mathbf{1 7}$ in low yield (Scheme 7). All the new compounds were characterized with ${ }^{1} \mathrm{H} N M R,{ }^{13} \mathrm{C}$ NMR, FTIR and LC-MSMS(Qtof) spectral data.

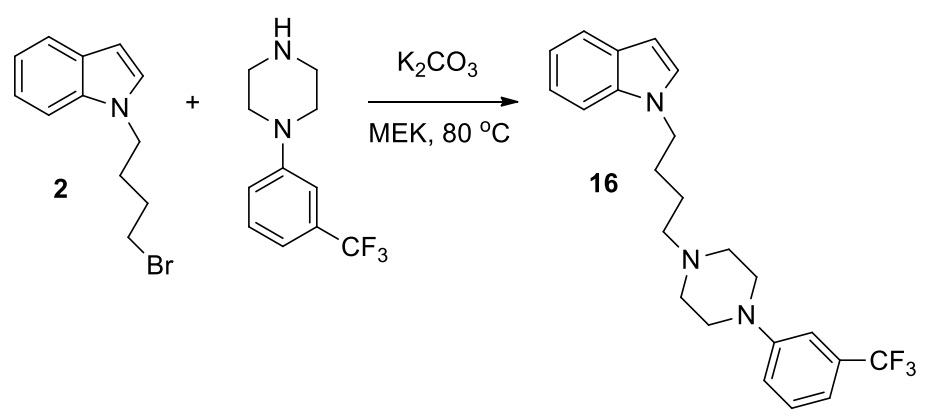

Scheme 6. Preparation of 16. 


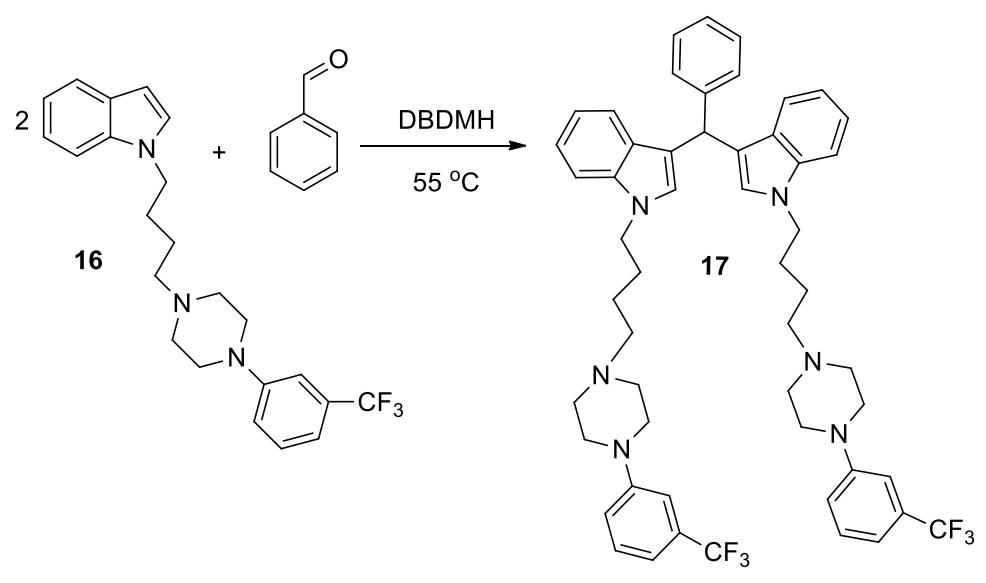

Scheme 7. Synthesis of 17.

\section{Conclusions}

It is well known that bisindoylmethane alkoloids have shown a wide spectrum of pharmacological activities that have been successfully screened for especially anti-cancer, antimicrobial, anti-inflammatory, antiviral and anti-leishmanial activities. On the other hand, aryl piperazines themselves are currently used as medicines and exhibit a wide range of activity in combination with various groups. Herein, we combined two important groups, bisindolylmethane and aryl piperazine with a hydrophobic chain for future activity studies. We anticipate significant biological activity from these substrates.

\section{Experimental Section}

General. All reagents were purchased from Aldrich or Merck and were used without further purification. The solvents were dried and distilled according to standard procedures. All melting points were determined on a Gallenkamp digital thermometer, are uncorrected. Reactions were monitored using TLC. Visualizations of the chromatograms were performed either with UV light or vanillin stain.

IR spectra were obtained with a Perkin Elmer Spectrum One FTIR Spectrometer and are reported in terms of the frequency of absorption $\left(\mathrm{cm}^{-1}\right) .{ }^{1} \mathrm{H} N M R$ and ${ }^{13} \mathrm{C}$ NMR spectra were recorded on a Bruker Avance III-500 MHz NMR spectrometer relative to tetramethylsilane $(\delta=0.00 \mathrm{ppm})$, with coupling constant $(J)$ values in Hertz $(\mathrm{Hz})$. Peak multiplicities are designated by the following abbreviations: s, singlet; d, doublet; dd, double doublet; $t$, triplet; $p t$, pentet; $d t$, double triplet; $\mathrm{m}$, multiplet; br, broad. Mass spectra were measured on an Agilent 6890N/5973 GC/IMSD system or LCMS 6400 Series Triple Quadrupole B.08.02 (B8260.0). Highresolution mass spectra were acquired in the positive ion mode using an Agilent G6530B TOF/Qtof Mass spectrometer.

General procedure for synthesis of compounds (3 - 6). Compound 2 ( $2 \mathrm{mmol})$, aromatic aldehyde ( $1 \mathrm{mmol})$ ( $p$-chlorobenzaldehyde, $p$-fluorobenzaldehyde, phenanthrene-9-carboxaldehyde or 2-naphthaldehyde, respectively) and 1,3-dibromo-5,5-dimethyl hydantoin (DBDMH) (0.005 mmol) were stirred at $55{ }^{\circ} \mathrm{C}$ for 16 hours without any solvent. After completion of the reaction with TLC control, crude products were purified by column chromatography. 
3,3'-((4-Chlorophenyl)methylene)bis(1-(4-bromobutyl)-1H-indole) (3). Pink solid (438 $\mathrm{mg}, 70 \%$ isolated yield). $\mathrm{mp} 50{ }^{\circ} \mathrm{C} . \mathrm{R}_{\mathrm{f}}=0.58\left(1: 5\right.$, ethyl acetate/n-hexane). FTIR $\left(\mathrm{KBr}, \mathrm{cm}^{-1}\right): 3046,2937,2872,1611(\mathrm{C}=\mathrm{C}), 1547(\mathrm{C}=\mathrm{C})$, $1487,1465,1088,798,736,702 .{ }^{1} \mathrm{H} N M R\left(\mathrm{CDCl}_{3}, 500 \mathrm{MHz}\right): \delta_{\mathrm{H}} 1.74-1.80\left(4 \mathrm{H}, \mathrm{m}, \mathrm{CH}_{2}\right), 1.89-1.95\left(4 \mathrm{H}, \mathrm{m}, \mathrm{CH}_{2}\right)$, $3.31\left(4 \mathrm{H}, \mathrm{t}, J 6.62 \mathrm{~Hz}, \mathrm{CH}_{2}\right), 4.05\left(4 \mathrm{H}, \mathrm{dt}, J 2.52\right.$ and J $\left.6.62 \mathrm{~Hz}, \mathrm{CH}_{2}\right), 5.83(1 \mathrm{H}, \mathrm{s}, \mathrm{CH}), 6.51(2 \mathrm{H}, \mathrm{s},=\mathrm{CH}), 6.99(2 \mathrm{H}$, $\mathrm{dt}, J 0.94$ and $J 7.88 \mathrm{~Hz}, \mathrm{ArH}), 7.18(2 \mathrm{H}, \mathrm{dt}, J 0.94$ and J $7.88 \mathrm{~Hz}, \mathrm{ArH}), 7.24-7.26(4 \mathrm{H}, \mathrm{m}, \operatorname{ArH}), 7.31(4 \mathrm{H}, \mathrm{dd}, J$ 8.19 and $J 12.29 \mathrm{~Hz}, \mathrm{ArH}) .{ }^{13} \mathrm{C} \mathrm{NMR}\left(\mathrm{CDCl}_{3}, 125 \mathrm{MHz}\right): \delta_{\mathrm{C}} 28.7\left(2 \times \mathrm{CH}_{2}\right), 29.9\left(2 \times \mathrm{CH}_{2}\right), 32.9\left(2 \times \mathrm{CH}_{2}\right), 39.5(\mathrm{CH})$, $45.3\left(2 \times \mathrm{CH}_{2}\right), 109.2$ (2xCAr), 117.9 (CAr), 118.8 (CAr), 120.0 (2xCAr), 121.6 (CAr), 127.0 (4xC), 127.4 (CAr), 128.3 (2x CAr), 130.0 (2x CAr), 131.7 (2xC), 131.8 (C) 136.6 (2xCAr), 142.7 (C). HRMS for $\mathrm{C}_{31} \mathrm{H}_{31} \mathrm{Br}_{2} \mathrm{ClN}_{2}$ calculated: 626.8522; Found $627.0408[\mathrm{M}+\mathrm{H}]^{+}$.

3,3'-((4-Fluorophenyl)methylene)bis(1-(4-bromobutyl)-1H-indole) (4). Dark pink solid (372 mg, 61\% isolated yield). $\mathrm{mp} 53^{\circ} \mathrm{C} . \mathrm{R}_{\mathrm{f}}=0.56$ (1:3, ethyl acetate/n-hexane). FTIR ( $\left.\mathrm{KBr}, \mathrm{cm}^{-1}\right): 3046,2922,2851,1601$ (C=C), 1547 (C=C) , 1504, 1479, 1465, 1092, 805, 783, 737. ${ }^{1} \mathrm{H} \mathrm{NMR}\left(\mathrm{CDCl}_{3}, 500 \mathrm{MHz}\right): \delta_{\mathrm{H}} 1.78\left(4 \mathrm{H}, \mathrm{pt}, J 6.93 \mathrm{~Hz}, \mathrm{CH}_{2}\right), 1.92$ $\left(4 \mathrm{H}, \mathrm{pt}, J 6.93 \mathrm{~Hz}, \mathrm{CH}_{2}\right), 3.31\left(4 \mathrm{H}, \mathrm{t}, J 6.62 \mathrm{~Hz}, \mathrm{CH}_{2}\right), 4.04\left(4 \mathrm{H}, \mathrm{dt}, J 2.20\right.$ and J $\left.6.62 \mathrm{~Hz}, \mathrm{CH}_{2}\right), 5.84(1 \mathrm{H}, \mathrm{s}, \mathrm{CH}), 6.51$ $(2 \mathrm{H}, \mathrm{s},=\mathrm{CH}), 6.94-7.00(4 \mathrm{H}, \mathrm{m}, \mathrm{ArH}), 7.18(2 \mathrm{H}, \mathrm{t}, J 7.25 \mathrm{~Hz}, \mathrm{ArH}), 7.27(2 \mathrm{H}, \mathrm{dt}, J 2.20$ and J 5.67 Hz, $\mathrm{ArH}), 7.30$ $(2 \mathrm{H}, \mathrm{d}, J 8.19 \mathrm{~Hz}, \mathrm{ArH}), 7.33(2 \mathrm{H}, \mathrm{d}, J 7.88 \mathrm{~Hz}, \mathrm{ArH}) .{ }^{13} \mathrm{C} \mathrm{NMR}\left(\mathrm{CDCl}_{3}, 125 \mathrm{MHz}\right): \delta_{\mathrm{C}} 28.7\left(2 \times \mathrm{CH}_{2}\right), 29.9\left(2 \times \mathrm{CH}_{2}\right)$, $32.9\left(2 \times \mathrm{CH}_{2}\right), 39.4(\mathrm{CH}), 45.3\left(2 \times \mathrm{CH}_{2}\right), 109.2(2 \times C \mathrm{Ar}), 114.8(\mathrm{CAr}), 115.0(\mathrm{CAr}), 118.3(\mathrm{C}), 118.8(2 \times C \mathrm{Ar}) 120.1$ (2xCAr), 121.6 (2xCAr), 127.0 (2xCAr), 127.4 (2xC), 129.9 (CAr), 130.0 (CAr), 136.6 (2xC), $139.8(C), 160.4(C)$, $162.3(C)$. LCMS for $\mathrm{C}_{31} \mathrm{H}_{31} \mathrm{Br}_{2} \mathrm{FN}_{2}$ calculated: 608.0837; Found $609.1000[\mathrm{M}+\mathrm{H}]^{+}$.

3,3'-(Phenanthren-9-ylmethylene)bis(1-(4-bromobutyl)-1H-indole) (5). Pink solid (393 $\mathrm{mg}, 57 \%$ isolated yield). $m p 89^{\circ} \mathrm{C} . R_{f}=0.45$ (1:5, ethyl acetate/n-hexane). FTIR $\left(K B r, \mathrm{~cm}^{-1}\right): 3015,2961,2921,2851,1654(\mathrm{C}=\mathrm{C})$, 1610 (C=C), 1545, 1465, 1446, 1081, 864, 796, 747, 728. ${ }^{1} \mathrm{H} \mathrm{NMR}\left(\mathrm{CDCl}_{3}, 500 \mathrm{MHz}\right): \delta_{\mathrm{H}} 1.69(4 \mathrm{H}, \mathrm{pt}, \mathrm{J} 6.62 \mathrm{~Hz}$, $\left.\mathrm{CH}_{2}\right), 1.884 \mathrm{H},\left(\mathrm{pt}, J 6.62 \mathrm{~Hz}, \mathrm{CH}_{2}\right), 3.25\left(4 \mathrm{H}, \mathrm{t}, J 6.62 \mathrm{~Hz}, \mathrm{CH}_{2}\right), 4.02\left(4 \mathrm{H}, \mathrm{t}, J 6.62 \mathrm{~Hz}, \mathrm{CH}_{2}\right), 6.49(2 \mathrm{H}, \mathrm{s},=\mathrm{CH}), 6.63$ $(1 \mathrm{H}, \mathrm{s}, \mathrm{CH}), 6.98(2 \mathrm{H}, \mathrm{dt}, J 0.94$ and $J 7.88 \mathrm{~Hz}, \mathrm{ArH}), 7.20(2 \mathrm{H}, \mathrm{dt}, J 0.94$ and $J 8.19 \mathrm{~Hz}, \mathrm{ArH}), 7.33(2 \mathrm{H}, \mathrm{d}, J 8.19 \mathrm{~Hz}$, $\operatorname{ArH}), 7.42(2 \mathrm{H}, \mathrm{d}, J 7.88 \mathrm{~Hz}, \mathrm{ArH}), 7.44-7.50(3 \mathrm{H}, \mathrm{m}, \mathrm{ArH}), 7.58-7.64(3 \mathrm{H}, \mathrm{m}, \mathrm{ArH}), 8.17(1 \mathrm{H}, \mathrm{d}, J 8.19 \mathrm{~Hz}, \mathrm{ArH})$, $8.69(1 \mathrm{H}, \mathrm{d}, J 8.19 \mathrm{~Hz}, \mathrm{ArH}), 8.76(1 \mathrm{H}, \mathrm{d}, J 8.19 \mathrm{~Hz}, \mathrm{ArH}) .{ }^{13} \mathrm{C} \mathrm{NMR}\left(\mathrm{CDCl}_{3}, 125 \mathrm{MHz}\right): \delta_{\mathrm{c}} 28.7\left(2 \times \mathrm{CH}_{2}\right), 29.7\left(\mathrm{CH}_{2}\right)$, $29.9\left(2 \times \mathrm{CH}_{2}\right), 32.9\left(2 \times \mathrm{CH}_{2}\right), 38.9(\mathrm{CH}), 45.2\left(\mathrm{CH}_{2}\right), 109.2(2 \times C \mathrm{Ar}), 117.7(2 \times C \mathrm{Ar}), 118.8(\mathrm{CAr}), 120.1$ (CAr), 121.5 (2xCAr), 122.3 (CAr), 122.9 (CAr), 125.1 (CAr), 125.9 (CAr), 126.1 (CAr), 126.3 (CAr), 126.5 (CAr), 126.7 (CAr), 127.7 (2xC), 128.0 (2xCAr), 128.7 (2xCAr), 129.5 (CAr), $129.8(C), 130.8(C), 131.3(C), 131.7(C), 136.7(2 \times C)$, $137.8(C)$. LCMS for $\mathrm{C}_{39} \mathrm{H}_{36} \mathrm{Br}_{2} \mathrm{~N}_{2}$ calculated: 690.1245 ; Found $691.1000[\mathrm{M}+\mathrm{H}]^{+}$.

3,3'-(Naphthalen-2-ylmethylene)bis(1-(4-bromobutyl)-1H-indole) (6). Light pink solid (531 mg, 83\% isolated yield). $m p 142{ }^{\circ} \mathrm{C} . R_{f}=0.45$ (1:5, ethyl acetate/ $n$-hexane). FTIR ( $\left.K B r, \mathrm{~cm}^{-1}\right): 3126,3048,2940,2877,1610$ (C=C), 1601 (C=C), 1549, 1478, 1466, 1444, 1434, 1096, 830, 804, 778, 766, 739. ${ }^{1} \mathrm{H} \mathrm{NMR}\left(\mathrm{CDCl}_{3}, 500 \mathrm{MHz}\right): \delta_{\mathrm{H}} 1.78$ $\left(4 \mathrm{H}, \mathrm{pt}, J 6.62 \mathrm{~Hz}, \mathrm{CH}_{2}\right), 1.92\left(4 \mathrm{H}, \mathrm{pt}, J 6.62 \mathrm{~Hz}, \mathrm{CH}_{2}\right), 3.31\left(4 \mathrm{H}, \mathrm{t}, J 6.62 \mathrm{~Hz}, \mathrm{CH}_{2}\right), 4.03-4.07(4 \mathrm{H}, \mathrm{m}, \mathrm{CH}), 6.03$ $(1 \mathrm{H}, \mathrm{s}, \mathrm{CH}), 6.54(2 \mathrm{H}, \mathrm{s},=\mathrm{CH}), 6.97(2 \mathrm{H}, \mathrm{dt}, J 0.94$ and $J 7.88 \mathrm{~Hz}, \mathrm{ArH}), 7.18(2 \mathrm{H}, \mathrm{dt}, J 0.94$ and $J 8.19 \mathrm{~Hz}, \mathrm{ArH})$, $7.31(2 \mathrm{H}, \mathrm{d}, J 8.19 \mathrm{~Hz}, \mathrm{ArH}), 7.39(2 \mathrm{H}, \mathrm{d}, J 7.88 \mathrm{~Hz}, \mathrm{ArH}), 7.42(1 \mathrm{H}, \mathrm{dd}, J 1.26$ and J $3.15 \mathrm{~Hz}, \operatorname{ArH}), 7.43(1 \mathrm{H}, \mathrm{d}, J$ $9.45 \mathrm{~Hz}, \mathrm{ArH}), 7.50(1 \mathrm{H}, \mathrm{dd}, J 1.57$ and $J 8.19 \mathrm{~Hz}, \operatorname{ArH}), 7.72-7.74(2 \mathrm{H}, \mathrm{m}, \operatorname{ArH}), 7.77(1 \mathrm{H}, \mathrm{d}, J 8.51 \mathrm{~Hz}, \operatorname{ArH})$, 7.80-7.82 (1H, m, ArH). ${ }^{13} \mathrm{C} \mathrm{NMR}\left(\mathrm{CDCl}_{3}, 125 \mathrm{MHz}\right): \delta_{\mathrm{c}} 28.7\left(2 \times \mathrm{CH}_{2}\right), 30.0\left(2 x \mathrm{CH}_{2}\right), 33.0\left(2 \times \mathrm{CH}_{2}\right), 40.2(\mathrm{CH}), 45.3$ (2xCH 2$), 109.1$ (2xCAr), 118.2 (2xCAr), 118.8 (2xCAr), 120.2 (2xCAr), 121.5 (2xCAr), 125.2 (CAr), 125.6 (CAr), 126.7 (CAr), 127.3 (CAr), 127.4 (CAr), 127.5 (CAr), 127.6 (2xCAr), 127.7 (2xCAr), 127.9 (CAr), 132.3 (2xC), 133.5 (C), $136.6(C), 141.7$ (C). LCMS for $\mathrm{C}_{35} \mathrm{H}_{34} \mathrm{Br}_{2} \mathrm{~N}_{2}$ calculated: 640.1088 ; Found $640.9000[\mathrm{M}+\mathrm{H}]^{+}$.

General procedure for synthesis of compounds $(7$ - 10). To a solution of the indole $(3 \mathrm{mmol})$ and aryl aldehyde $(1 \mathrm{mmol})$ in $\mathrm{MeCN}(2.5 \mathrm{~mL})$ was added a solution of $\mathrm{AuCl}_{3}(1 \mathrm{~mol} \%)$ in $\mathrm{MeCN}(1 \mathrm{~mL})$ under an atmosphere of nitrogen. After stirring the reaction mixture at r.t. for $12 \mathrm{~h}$, it was filtered to remove insoluble 
impurities. The residue was purified by silica gel column chromatography (100-200 mesh silica gel, hexaneEtOAc, 95:5) to afford the products.

General procedure for synthesis of compounds (11 - 13). A mixture of compound 3, 5 or 6 (1 mmol), 1-(2,3dichlorophenyl)piperazine hydrochloride $(2 \mathrm{mmol})$, and anhydrous $\mathrm{K}_{2} \mathrm{CO}_{3}(2 \mathrm{mmol})$ in $\mathrm{MEK}(50 \mathrm{~mL})$ was heated at $80{ }^{\circ} \mathrm{C}$ for $16 \mathrm{~h}$. After completion of the reaction with TLC control, the solvent was evaporated and the residue was purified by column chromatography using ethyl acetate/ $n$-hexane as the eluent to give pure products 11, 12 or 13.

\section{1-(4-Bromobutyl)-3-((4-chlorophenyl)(1-(4-(4-(2,3-dichlorophenyl)piperazin-1-yl)butyl)-1H-indol-3-}

yl)methyl)-1H-indole (11). Light pink solid (494 mg, $61 \%$ isolated yield). $\mathrm{mp} 57{ }^{\circ} \mathrm{C} . \mathrm{R}_{\mathrm{f}}=0.22$ (10:1, ethyl acetate/n-hexane). FTIR ( $\mathrm{KBr}, \mathrm{cm}^{-1}$ ): 3045, 2927, 2852, 2810, 1610 (C=C), 1583 (C=C), 1545, 1487, 1466, 1445, 1087, 795, 737. ${ }^{1} \mathrm{H}$ NMR $\left(\mathrm{CDCl}_{3}, 500 \mathrm{MHz}\right): \delta_{\mathrm{H}} 1.49\left(2 \mathrm{H}, \mathrm{pt}, J 6.93 \mathrm{~Hz}, \mathrm{CH}_{2}\right), 1.68-1.73\left(1 \mathrm{H}, \mathrm{m}, \mathrm{CH}_{2}\right), 1.76-1.85$ $\left(3 \mathrm{H}, \mathrm{m}, \mathrm{CH}_{2}\right), 1.92\left(2 \mathrm{H}, \mathrm{pt}, J 6.93 \mathrm{~Hz}, \mathrm{CH}_{2}\right), 2.38\left(2 \mathrm{H}, \mathrm{t}, J 7.56 \mathrm{~Hz}, \mathrm{CH}_{2}\right), 2.55\left(4 \mathrm{H}, \mathrm{brs}, \mathrm{CH}_{2}\right), 3.02\left(4 \mathrm{H}, \mathrm{brs}, \mathrm{CH}_{2}\right)$, $3.31\left(1 \mathrm{H}, \mathrm{t}, J 6.62 \mathrm{~Hz}, \mathrm{CH}_{2}\right), 4.05\left(5 \mathrm{H}, \mathrm{t}, J 6.93 \mathrm{~Hz}, \mathrm{CH}_{2}\right), 5.84(1 \mathrm{H}, \mathrm{s}, \mathrm{CH}), 6.53(2 \mathrm{H}, \mathrm{s},=\mathrm{CH}), 6.95(1 \mathrm{H}, \mathrm{dd}, J 2.52$ and J $6.93 \mathrm{~Hz}, \operatorname{ArH}), 6.99(2 \mathrm{H}, \mathrm{t}, J 7.25 \mathrm{~Hz}, \operatorname{ArH}), 7.15(2 \mathrm{H}, \mathrm{d}, J 7.88 \mathrm{~Hz}, \operatorname{ArH}), 7.18(2 \mathrm{H}, \mathrm{t}, J 7.25 \mathrm{~Hz}, \operatorname{ArH}), \quad 7.25$ $(4 \mathrm{H}, \mathrm{d}, J 3.46 \mathrm{~Hz}, \mathrm{ArH}), 7.30(1 \mathrm{H}, \mathrm{d}, J 8.19 \mathrm{~Hz}, \mathrm{ArH}), 7.32-7.35(3 \mathrm{H}, \mathrm{m}, \mathrm{ArH}) .{ }^{13} \mathrm{C} \mathrm{NMR}\left(\mathrm{CDCl}_{3}, 125 \mathrm{MHz}\right): \delta_{c} 24.1$ $\left(\mathrm{CH}_{2}\right), 28.1\left(\mathrm{CH}_{2}\right), 28.7\left(\mathrm{CH}_{2}\right), 29.6\left(\mathrm{CH}_{2}\right), 29.9\left(\mathrm{CH}_{2}\right), 32.9\left(\mathrm{CH}_{2}\right), 39.6(\mathrm{CH}), 44.3\left(\mathrm{CH}_{2}\right), 45.3\left(\mathrm{CH}_{2}\right), 46.1\left(\mathrm{CH}_{2}\right)$, $51.2\left(\mathrm{CH}_{2}\right), 53.1\left(\mathrm{CH}_{2}\right), 57.8\left(\mathrm{CH}_{2}\right), 109.2$ (CAr), 109.4 (CAr), $117.6(C), 117.9(C), 118.0(C), 118.5(C A r), 118.7$ (CAr), 118.8 (CAr), 120.0 (CAr), 120.1 (CAr), 121.4 (CAr), 121.6 (CAr), 124.5 (CAr), 127.0 (CAr), 127.2 (CAr), $127.3(C), 127.4$ (CAr), $127.5(C), 128.3$ (2xCAr), 130.0 (2xCAr), $131.7(C), 134.0(C), 136.6(C), 136.7(C), 142.8$ (C), $151.2(C)$. HRMS for $\mathrm{C}_{41} \mathrm{H}_{42} \mathrm{BrCl}_{3} \mathrm{~N}_{4}$ calculated: 774.1658 ; found: $775.1992[\mathrm{M}+\mathrm{H}]^{+}$.

\section{1-(4-Bromobutyl)-3-((1-(4-(4-(2,3-dichlorophenyl)piperazin-1-yl)butyl)-1H-indol-3-yl)(phenanthren-9-}

yl)methyl)- $\mathbf{H}$-indole (12). Light pink solid $\left(655 \mathrm{mg}, 77.9 \%\right.$ isolated yield). $\mathrm{mp} 76{ }^{\circ} \mathrm{C} . \mathrm{R}_{\mathrm{f}}=0.63$ (20:1, ethyl acetate/n-hexane). FTIR (KBr, cm$\left.{ }^{-1}\right)$ : 3052, 2922, 2850, 1611 (C=C), 1577 (C=C), 1465, 1448, 1421, 1043, 780, 740, 726, 713, 671. ${ }^{1} \mathrm{H} \mathrm{NMR}\left(\mathrm{CDCl}_{3}, 500 \mathrm{MHz}\right): \delta_{\mathrm{H}} 1.39\left(2 \mathrm{H}, \mathrm{pt}, J 7.56 \mathrm{~Hz}, \mathrm{CH}_{2}\right), 1.47\left(2 \mathrm{H}, \mathrm{pt}, J 6.30 \mathrm{~Hz}, \mathrm{CH}_{2}\right)$, 1.76-1.80 (6H, m, CH $\mathrm{CH}_{2}, 2.33\left(2 \mathrm{H}, \mathrm{t}, \mathrm{J} 7.56 \mathrm{~Hz}, \mathrm{CH}_{2}\right), 2.47-2.50\left(2 \mathrm{H}, \mathrm{m}, \mathrm{CH}_{2}\right), 2.96-2.99\left(2 \mathrm{H}, \mathrm{m}, \mathrm{CH}_{2}\right), 3.95(2 \mathrm{H}, \mathrm{t}, J$ $\left.6.30 \mathrm{~Hz}, \mathrm{CH}_{2}\right), 3.99-4.04\left(6 \mathrm{H}, \mathrm{m}, \mathrm{CH}_{2}\right), 6.50(1 \mathrm{H}, \mathrm{s},=\mathrm{CH}), 6.52(1 \mathrm{H}, \mathrm{s},=\mathrm{CH}), 6.64(1 \mathrm{H}, \mathrm{s}, \mathrm{CH}), 6.91(1 \mathrm{H}, \mathrm{dd}, J 2.20$ and J $7.25 \mathrm{~Hz}, \operatorname{ArH}), 6.97(2 \mathrm{H}, \mathrm{t}, J 7.88 \mathrm{~Hz}, \operatorname{ArH}), 7.15(2 \mathrm{H}, \mathrm{d}, J 2.20 \mathrm{~Hz}, \mathrm{ArH}), 7.19(2 \mathrm{H}, \mathrm{ddd}, J 2.20$ and $J 4.49$ and J $9.77 \mathrm{~Hz}, \operatorname{ArH}), 7.32(1 \mathrm{H}, \mathrm{d}, J 8.19 \mathrm{~Hz}, \operatorname{ArH}), 7.35(1 \mathrm{H}, \mathrm{d}, J 8.19 \mathrm{~Hz}, \mathrm{ArH}), 7.41(2 \mathrm{H}, \mathrm{dd}, J 3.78 \mathrm{and} J 7.78 \mathrm{~Hz}, \operatorname{ArH})$, 7.44-7.48 (3H, m, ArH), 7.57-7.62 (3H, m, ArH), $8.18(1 \mathrm{H}, \mathrm{d}, J 8.19 \mathrm{~Hz}, \mathrm{ArH}), 8.68(1 \mathrm{H}, \mathrm{d}, J 8.51 \mathrm{~Hz}, \mathrm{ArH}), 8.75$ $(1 \mathrm{H}, \mathrm{d}, J 8.19 \mathrm{~Hz}, \mathrm{ArH}) .{ }^{13} \mathrm{C} \mathrm{NMR}\left(\mathrm{CDCl}_{3}, 125 \mathrm{MHz}\right): \delta_{\mathrm{c}} 25.8\left(\mathrm{CH}_{2}\right), 26.7\left(2 \mathrm{xCH}_{2}\right), 28.0\left(\mathrm{CH}_{2}\right), 29.7\left(2 x \mathrm{CH}_{2}\right), 36.0$ $(\mathrm{CH}), 45.7\left(\mathrm{CH}_{2}\right), 46.0\left(\mathrm{CH}_{2}\right), 51.0\left(\mathrm{CH}_{2}\right), 53.0\left(\mathrm{CH}_{2}\right), 57.8\left(\mathrm{CH}_{2}\right), 63.8\left(\mathrm{CH}_{2}\right), 109.2(\mathrm{CAr}), 109.3(\mathrm{CAr}), 117.3(\mathrm{C})$, 117.6 (C), 118.5 (CAr), 118.6 (CAr), 118.7 (CAr), 120.0 (2xCAr), 121.3 (CAr), 121.4 (CAr), 122.3 (CAr), 122.9 (CAr), 124.6 (CAr), 125.2 (CAr), 125.9 (CAr), 126.1 (CAr), 126.2 (CAr), 126.5 (CAr), 126.7 (CAr), 127.4 (CAr), $127.6(2 \times C), 127.7(C), 128.1$ (2xCAr), 128.7 (CAr), $129.8(2 \times C), 130.8(C), 131.3(C), 131.7(C), 134.0(C), 136.7$ (2xC), $137.9(C)$. HRMS for $\mathrm{C}_{49} \mathrm{H}_{47} \mathrm{BrCl}_{2} \mathrm{~N}_{4}$ calculated: 842.7343 ; found: $843.2392[\mathrm{M}+\mathrm{H}]^{+}$.

\section{1-(4-Bromobutyl)-3-((1-(4-(4-(2,3-dichlorophenyl)piperazin-1-yl)butyl)-1H-indol-3-yl)(naphthalen-2-}

yl)methyl)-1H-indole (13). White solid (641.5 g, $81 \%$ isolated yield). $\mathrm{mp} 87^{\circ} \mathrm{C} . \mathrm{R}_{\mathrm{f}}=0.30$ (8:1, ethanol/nhexane) FTIR ( $\left.\mathrm{KBr}, \mathrm{cm}^{-1}\right)$ : 3001, 2926, 2850, 1572 (C=C), 1408, 1012, 738, 673, 646. ${ }^{1} \mathrm{H} \mathrm{NMR}\left(\mathrm{CDCl}_{3}, 500 \mathrm{MHz}\right)$ : $\delta_{\mathrm{H}} 1.47\left(4 \mathrm{H}, \mathrm{pt}, J 7.56\right.$ and $\left.J 14.81 \mathrm{~Hz}, \mathrm{CH}_{2}\right), 1.81\left(4 \mathrm{H}, \mathrm{pt}, J 7.56\right.$ and $\left.J 14.81 \mathrm{~Hz}, \mathrm{CH}_{2}\right), 2.36\left(4 \mathrm{H}, \mathrm{t}, J 7.56 \mathrm{~Hz}, \mathrm{CH}_{2}\right)$, $2.52\left(6 \mathrm{H}\right.$, brs, $\left.\mathrm{CH}_{2}\right), 2.99\left(6 \mathrm{H}, \mathrm{brs}, \mathrm{CH}_{2}\right), 6.03(1 \mathrm{H}, \mathrm{s}, \mathrm{CH}), 6.57(2 \mathrm{H}, \mathrm{s},=\mathrm{CH}), 6.92(1 \mathrm{H}, \mathrm{dd}, J 2.20 \mathrm{and} J 6.93 \mathrm{~Hz}$, $\operatorname{ArH}), 6.95(2 \mathrm{H}, \mathrm{t}, J 7.56 \mathrm{~Hz}, \operatorname{ArH}), 7.12-7.18(6 \mathrm{H}, \mathrm{m}, \mathrm{ArH}), 7.32(2 \mathrm{H}, \mathrm{d}, J 8.19 \mathrm{~Hz}, \operatorname{ArH}), 7.38-7.40(3 \mathrm{H}, \mathrm{m}, \mathrm{ArH})$, 7.49-7.51 (1H, m, ArH), 7.70-7.72 (1H, m, ArH), 7.74-7.77 (1H, m, ArH), 7.78-7.80 (1H, m, ArH). ${ }^{13} \mathrm{C} \mathrm{NMR}$ $\left(\mathrm{CDCl}_{3}, 125 \mathrm{MHz}\right): \delta_{\mathrm{c}} 24.1\left(\mathrm{CH}_{2}\right), 28.1\left(2 x \mathrm{CH}_{2}\right), 29.6\left(\mathrm{CH}_{2}\right), 40.3(\mathrm{CH}), 46.1\left(\mathrm{CH}_{2}\right), 51.2\left(4 \times \mathrm{CH}_{2}\right), 53.1\left(2 x C \mathrm{H}_{2}\right), 57.9$ $\left(\mathrm{CH}_{2}\right), 109.2$ (CAr), $117.9(2 \times C), 118.5$ (2xCAr), 118.6 (CAr), 120.1 (2xCAr), 121.3 (2xCAr), 124.5 (CAr), 125.2 
(CAr), 125.6 (CAr), 126.6 (CAr), 127.4 (2xCAr), 127.5 (CAr), 127.6 (2xC), 127.7 (CAr), 127.8 (CAr), 127.9 (CAr), 128.2 (CAr), 130.0 (CAr), 132.2 (C), $133.5(C), 133.9$ (C), 136.6 (2xC), $141.9(C), 151.2$ (2xC). HRMS for $\mathrm{C}_{45} \mathrm{H}_{45} \mathrm{BrCl}_{2} \mathrm{~N}_{4}$ calculated: 792.6756; found: $793.2226[\mathrm{M}+\mathrm{H}]^{+}$.

General procedure for synthesis of compounds (14 and 15). A mixture of compound 3 or 4 (1 mmol), 2(piperazin-1-yl)pyrimidine (2 mmol), and anhydrous $\mathrm{K}_{2} \mathrm{CO}_{3}(2 \mathrm{mmol})$ in MEK $(50 \mathrm{~mL})$ was heated at $80{ }^{\circ} \mathrm{C}$ for 20 h. After completion of the reaction with TLC control, the solvent was evaporated and the residue was purified by column chromatography using ethyl acetate/n-hexane (30:1) as the eluent to give pure products 14 or 15 .

1-(4-Bromobutyl)-3-((4-chlorophenyl)(1-(4-(4-(pyrimidin-2-yl)piperazin-1-yl)butyl)-1H-indol-3-yl)methyl)-1Hindole (14). Red solid (372 mg, 52.6\% isolated yield). $\mathrm{mp} 64{ }^{\circ} \mathrm{C}$. $\mathrm{R}_{\mathrm{f}}=0.22$ (10:1, ethyl acetate/ $n$-hexane). FTIR $\left(\mathrm{KBr}, \mathrm{cm}^{-1}\right)$ : FTIR $\left(\mathrm{KBr}, \mathrm{cm}^{-1}\right)$ : 3045, 2927, 2852, 2810, 1610 (C=C), 1583 (C=C), 1545, 1487, 1466, 1445, 1087, 795, 737. ${ }^{1} \mathrm{H} \mathrm{NMR}\left(\mathrm{CDCl}_{3}, 500 \mathrm{MHz}\right): \delta_{\mathrm{H}} 1.49\left(2 \mathrm{H}, \mathrm{pt}, J 7.56 \mathrm{~Hz}, \mathrm{CH}_{2}\right), 1.57\left(2 \mathrm{H}, \mathrm{pt}, J 7.56 \mathrm{~Hz}, \mathrm{CH}_{2}\right), 1.81(4 \mathrm{H}, \mathrm{pt}, J$ $\left.7.56 \mathrm{~Hz}, \mathrm{CH}_{2}\right), 2.34\left(2 \mathrm{H}, \mathrm{t}, J 7.56 \mathrm{~Hz}, \mathrm{CH}_{2}\right), 2.42\left(4 \mathrm{H}, \mathrm{t}, J 5.04 \mathrm{~Hz}, \mathrm{CH}_{2}\right), 3.80\left(4 \mathrm{H}, \mathrm{t}, J 4.41 \mathrm{~Hz}, \mathrm{CH}_{2}\right), 4.00-4.06(6 \mathrm{H}$, $\left.\mathrm{m}, \mathrm{CH}_{2}\right), 5.83(1 \mathrm{H}, \mathrm{s}, \mathrm{CH}), 6.48(1 \mathrm{H}, \mathrm{t}, J 4.72 \mathrm{~Hz}, \mathrm{ArH}), 6.52(2 \mathrm{H}, \mathrm{s},=\mathrm{CH}), 6.90(2 \mathrm{H}, \mathrm{dt}, J 2.83 \mathrm{and} J 7.56 \mathrm{~Hz}, \mathrm{ArH})$, $7.11(2 \mathrm{H}, \mathrm{dt}, J 1.26$ and $J 8.51 \mathrm{~Hz}, \mathrm{ArH}), 7.17(4 \mathrm{H}, \mathrm{brd}, J 1.26 \mathrm{~Hz}, \operatorname{ArH}), 7.23-7.25(4 \mathrm{H}, \mathrm{m}, \operatorname{ArH}), 8.30(2 \mathrm{H}, \mathrm{d}, J$ $4.72 \mathrm{~Hz}, \mathrm{ArH}) .{ }^{13} \mathrm{C} \mathrm{NMR}\left(\mathrm{CDCl}_{3}, 125 \mathrm{MHz}\right): \delta_{\mathrm{c}} 23.9\left(\mathrm{CH}_{2}\right), 26.0\left(2 \times \mathrm{CH}_{2}\right), 26.8\left(\mathrm{CH}_{2}\right), 28.0\left(\mathrm{CH}_{2}\right), 29.6\left(\mathrm{CH}_{2}\right), 39.6$ $(\mathrm{CH}), 43.4\left(\mathrm{CH}_{2}\right), 45.7\left(\mathrm{CH}_{2}\right), 46.0\left(\mathrm{CH}_{2}\right), 52.9\left(\mathrm{CH}_{2}\right), 57.9\left(\mathrm{CH}_{2}\right), 63.8\left(\mathrm{CH}_{2}\right), 109.2(\mathrm{CAr}), 109.3(\mathrm{CAr}), 109.8(\mathrm{CAr})$, 117.6 (C), $117.8(C), 118.6$ (CAr), 118.7 (CAr), 120.0 (2x CAr), 121.4 (CAr), 121.5 (CAr), 127.1 (CAr), 127.2 (CAr), $127.3(C), 127.4(C), 128.3$ (2xCAr), 130.0 (2xCAr), $131.6(C), 136.6(2 \times C), 142.8(C), 157.7(2 \times C A r), 161.56(C)$. HRMS for $\mathrm{C}_{39} \mathrm{H}_{42} \mathrm{BrClN}_{6}$ calculated: 708.2369; found: $747.2048[\mathrm{M}+\mathrm{K}]^{+}$.

1-(4-Bromobutyl)-3-((4-fluorophenyl)(1-(4-(4-(pyrimidin-2-yl)piperazin-1-yl)butyl)-1H-indol-3-yl)methyl)-1Hindole (15). Yellow solid ( $323 \mathrm{mg}, 46.6 \%$ isolated yield). $\mathrm{mp} 84^{\circ} \mathrm{C}$. $\mathrm{R}_{\mathrm{f}}=0.25$ (12:1, ethyl acetate/ $n$-hexane) FTIR $\left(\mathrm{KBr}, \mathrm{cm}^{-1}\right)$ : 3045, 2932, 2853, 2810, 1583 (C=C), 1546 (C=C), 1504, 1480, 1466, 1445, 1043, 834, 795, 737. ${ }^{1} \mathrm{H}$ $\mathrm{NMR}\left(\mathrm{CDCl}_{3}, 500 \mathrm{MHz}\right): \delta_{\mathrm{H}} 1.48\left(2 \mathrm{H}, \mathrm{pt}, J 7.33 \mathrm{~Hz}, \mathrm{CH}_{2}\right), 1.56\left(2 \mathrm{H}, \mathrm{pt}, J 6.84 \mathrm{~Hz}, \mathrm{CH}_{2}\right), 1.81(4 \mathrm{H}, \mathrm{pt}, J 6.84 \mathrm{~Hz}$, $\left.\mathrm{CH}_{2}\right), 2.33\left(2 \mathrm{H}, \mathrm{t}, J 7.33 \mathrm{~Hz}, \mathrm{~N}-\mathrm{CH}_{2}\right), 2.40\left(4 \mathrm{H}, \mathrm{t}, J 5.38 \mathrm{~Hz}, \mathrm{~N}-\mathrm{CH}_{2}\right), 3.78\left(4 \mathrm{H}, \mathrm{t}, J 5.38 \mathrm{~Hz}, \mathrm{~N}-\mathrm{CH}_{2}\right), 4.01(2 \mathrm{H}, \mathrm{t}, J$ $\left.6.84 \mathrm{~Hz}, \mathrm{~N}-\mathrm{CH}_{2}\right), 4.04\left(4 \mathrm{H}, \mathrm{t}, J 6.84 \mathrm{~Hz}, \mathrm{~N}-\mathrm{CH}_{2}\right), 5.84(1 \mathrm{H}, \mathrm{s}, \mathrm{CH}), 6.47(1 \mathrm{H}, \mathrm{t}, J 4.89 \mathrm{~Hz}, \mathrm{ArH}), 6.52(2 \mathrm{H}, \mathrm{brd}, J 1.95$ $\mathrm{Hz},=\mathrm{CH}), 6.94-6.99(4 \mathrm{H}, \mathrm{m}, \mathrm{ArH}), 7.17(2 \mathrm{H}, \mathrm{t}, J 7.82 \mathrm{~Hz}, \mathrm{ArH}), 7.28(2 \mathrm{H}, \mathrm{dd}, J 2.93 \mathrm{and} J 8.31 \mathrm{~Hz}, \operatorname{ArH}), 7.32-7.36$ $(4 \mathrm{H}, \mathrm{m}, \mathrm{ArH}), 8.32(2 \mathrm{H}, \mathrm{d}, \mathrm{J} 4.89 \mathrm{~Hz}, \mathrm{ArH}) .{ }^{13} \mathrm{C} \mathrm{NMR}\left(\mathrm{CDCl}_{3}, 125 \mathrm{MHz}\right): \delta_{\mathrm{c}} 24.0\left(\mathrm{CH}_{2}\right), 26.0\left(\mathrm{CH}_{2}\right), 26.8\left(\mathrm{CH}_{2}\right), 28.1$ $\left(\mathrm{CH}_{2}\right)$, $39.5(\mathrm{CH}), 43.6\left(2 x \mathrm{CH}_{2}\right), 45.7\left(\mathrm{CH}_{2}\right), 46.0\left(\mathrm{CH}_{2}\right), 53.0\left(\mathrm{CH}_{2}\right), 58.0\left(\mathrm{CH}_{2}\right), 63.8\left(2 x \mathrm{CH}_{2}\right), 109.2(\mathrm{CAr}), 109.3$ (CAr), 109.8 (CAr), 114.8 (CAr), 114.9 (CAr), 118.0 (C), 118.2 (C), 118.6 (CAr), 118.7 (CAr), 120.1 (CAr), 120.2 (CAr), 121.4 (CAr), 121.5 (CAr), 127.1 (CAr), 127.2 (CAr), 127.4 (C), 127.5 (C), 129.9 (CAr), 130.0 (CAr), 136.6 (C), $139.9(C), 157.6$ (2xCAr), $160.3(C), 161.6(C), 162.3(C)$. HRMS for $\mathrm{C}_{39} \mathrm{H}_{42} \mathrm{BrFN}_{6}$ calculated: 693.2675; found: $694.2655[\mathrm{M}+\mathrm{H}]^{+}$.

Synthesis of 1-(4-(4-(3-(Trifluoromethyl)phenyl)piperazin-1-yl)butyl)-1H-indole (16). A mixture of compound 2 (252.15 mg, $1 \mathrm{mmol})$, 1-(3-(trifluoromethyl)phenyl)piperazine $\left(535.2 \mathrm{mg}, 1 \mathrm{mmol}\right.$ ), and anhydrous $\mathrm{K}_{2} \mathrm{CO}_{3}$ $(276 \mathrm{mg}, 2 \mathrm{mmol})$ in MEK $(10 \mathrm{~mL})$ was heated at $80^{\circ} \mathrm{C}$ for $16 \mathrm{~h}$. After completion of the reaction with TLC control, the solvent was evaporated and the residue was purified by column chromatography using ethyl acetate/n-hexane (1:1) as the eluent to give pure product 16 [15]. White solid (300 mg, 75\% isolated yield). bp $523{ }^{\circ} \mathrm{C} . \mathrm{R}_{\mathrm{f}}=0.36\left(1: 1\right.$, ethyl acetate/n-hexane) $\mathrm{FTIR}\left(\mathrm{KBr}, \mathrm{cm}^{-1}\right): 3099,3053,2953,2873,1611,1515,1484$, 1463, 1084 (C-N), 763, 738, 718. ${ }^{1} \mathrm{H}$ NMR $\left(\mathrm{CDCl}_{3}, 500 \mathrm{MHz}\right): \delta_{\mathrm{H}} 1.57\left(2 \mathrm{H}, \mathrm{pt}, J 7.25 \mathrm{~Hz}, \mathrm{CH}_{2}\right), 1.90(2 \mathrm{H}, \mathrm{pt}, J 7.25$ $\left.\mathrm{Hz}, \mathrm{CH}_{2}\right), 2.42\left(2 \mathrm{H}, \mathrm{t}, J 7.56 \mathrm{~Hz}, \mathrm{CH}_{2}\right), 2.58\left(4 \mathrm{H}, \mathrm{t}, J 5.04 \mathrm{~Hz}, \mathrm{CH}_{2}\right), 3.22\left(4 \mathrm{H}, \mathrm{t}, J 5.04 \mathrm{~Hz}, \mathrm{CH}_{2}\right), 4.17(2 \mathrm{H}, \mathrm{t}, J 7.25$ $\left.\mathrm{Hz}, \mathrm{CH}_{2}\right), 6.49(1 \mathrm{H}, \mathrm{dd}, J 0.94$ and J $3.15 \mathrm{~Hz}, \mathrm{ArH}), 7.04(1 \mathrm{H}, \mathrm{dd}, J 2.52$ and J $8.51 \mathrm{~Hz}, \mathrm{ArH}), 7.07-7.12(4 \mathrm{H}, \mathrm{m}, \mathrm{ArH})$, $7.21(1 \mathrm{H}$, ddd, J 1.26 and J 8.19 and $J 15.44 \mathrm{~Hz}, \operatorname{ArH}), 7.32-7.36(2 \mathrm{H}, \mathrm{m}, \mathrm{ArH}), 7.64(1 \mathrm{H}, \mathrm{d}, J 7.88 \mathrm{~Hz}, \operatorname{ArH})$. GC-MS (El, $70 \mathrm{eV}): \mathrm{C}_{23} \mathrm{H}_{26} \mathrm{~F}_{3} \mathrm{~N}_{3} \mathrm{~m} / \mathrm{z}=401[\mathrm{M}]^{+}$. 


\section{Synthesis of 3,3'-(Phenylmethylene)bis(1-(4-(4-(3-8trifluoromethyl)phenyl)piperazin-1-yl)butyl)-1H-indole}

(17). Compound 16 ( $2 \mathrm{mmol})$, benzaldehyde $(1 \mathrm{mmol})$ and 1,3-dibromo-5,5-dimethyl hydantoin (DBDMH) $(0.005 \mathrm{mmol})$ were stirred at $55^{\circ} \mathrm{C}$ for 16 hours without any solvent. After completion of the reaction with TLC control, the solvent was evaporated and the residue was purified by column chromatography using ethyl acetate/ $n$-hexane (1:1) as the eluent to give pure product 17. Light pink solid (428 $\mathrm{mg}, 48 \%$ isolated yield). $\mathrm{mp}$ $73{ }^{\circ} \mathrm{C} . \mathrm{R}_{\mathrm{f}}=0.18\left(5: 1\right.$, ethyl acetate $/ n$-hexane). FTIR $\left(\mathrm{KBr}, \mathrm{cm}^{-1}\right): 3015,2966,2923,2882,2853,1609(\mathrm{C}=\mathrm{C})$, 1494, 1466, 1452, 1098, 782, 740, 695. ${ }^{1} \mathrm{H} \mathrm{NMR}\left(\mathrm{CDCl}_{3}, 500 \mathrm{MHz}\right): \delta_{\mathrm{H}} 1.47\left(4 \mathrm{H}, \mathrm{pt}, J 7.56 \mathrm{~Hz}, \mathrm{CH}_{2}\right), 1.81(4 \mathrm{H}, \mathrm{pt}$, J $\left.7.56 \mathrm{~Hz}, \mathrm{CH}_{2}\right), 2.34\left(4 \mathrm{H}, \mathrm{t}, J 7.56 \mathrm{~Hz}, \mathrm{CH}_{2}\right), 2.48\left(8 \mathrm{H}, \mathrm{t}, J 5.04 \mathrm{~Hz}, \mathrm{CH}_{2}\right), 3.16\left(8 \mathrm{H}, \mathrm{t}, J 5.04 \mathrm{~Hz}, \mathrm{CH}_{2}\right), 4.04(4 \mathrm{H}, \mathrm{t}, J$ $\left.6.93 \mathrm{~Hz}, \mathrm{CH}_{2}\right), 5.87(1 \mathrm{H}, \mathrm{s}, \mathrm{CH}), 6.56(2 \mathrm{H}, \mathrm{s},=\mathrm{CH}), 6.96(2 \mathrm{H}, \mathrm{dt}, J 0.94$ and $J 7.88 \mathrm{~Hz}, \operatorname{ArH}), 7.02(2 \mathrm{H}, \mathrm{dd}, J 2.20$ and J $8.51 \mathrm{~Hz}, \operatorname{ArH}), 7.06-7.08(4 \mathrm{H}, \mathrm{m}, \mathrm{ArH}), 7.15-7.21(\mathrm{m3H}, \mathrm{ArH}), 7.25-7.28(3 \mathrm{H}, \mathrm{m}, \mathrm{ArH}), 7.30-7.37(7 \mathrm{H}, \mathrm{m}, \mathrm{ArH})$.

${ }^{13} \mathrm{C} \mathrm{NMR}\left(\mathrm{CDCl}_{3}, 125 \mathrm{MHz}\right): \delta_{\mathrm{c}} 24.0\left(2 \times \mathrm{CH}_{2}\right), 28.0\left(2 \times \mathrm{CH}_{2}\right), 40.2(\mathrm{CH}), 46.0\left(2 \mathrm{xCH}_{2}\right), 48.5\left(4 \times \mathrm{CH}_{2}\right), 52.8\left(4 \times \mathrm{CH}_{2}\right)$, $57.8\left(2 \times \mathrm{CH}_{2}\right.$ ), 109.2 (2xCAr), 112.0 (CAr), 112.1 (CAr), 115.7 (CAr), 115.8 (CAr), 118.2 (2xC), 118.6 (4xCAr), 120.2 (2xCAr), 121.3 (2xCAr), 126.0 (CAr), 127.2 (2xCAr), 127.6 (2xC), 128.1 (2xCAr), 128.7 (2xCAr), 129.5 (4xCAr), $131.2(C), 131.5(C), 132.5(C), 136.6(2 \times C), 144.3(C), 151.3(C)$. HRMS for $\mathrm{C}_{53} \mathrm{H}_{56} \mathrm{~F}_{6} \mathrm{~N}_{6}$ calculated: 891.4548; found: $892.4575[\mathrm{M}+\mathrm{H}]^{+}$.

\section{Acknowledgements}

The authors gratefully acknowledge the financial support of this work by the Ministry of Science, Industry and Technology (SANTEZ, Project No. 0048.STZ.2013-1).

\section{Supplementary Material}

New bisindolylmethanes list and IR, ${ }^{1} \mathrm{H}-\mathrm{NMR},{ }^{13} \mathrm{C}-\mathrm{NMR}$, Qtof or LCMS spectra of new compounds.

\section{References}

1. Imran, S.; Taha, M.; Ismail, N. H.; Fayyaz, S.; Khan, K. M.; Choudhary, M.I. Bioorganic Chem. 2016, 68, 90104.

https://doi.org/10.1016/j.bioorg.2016.07.011

2. Siadatifard, S. H.; Abdoli-Senejani, M.; Bodaghifard, M. A. Cogent Chem. 2016, 2, UNSP 1188435.

3. Samuel, R. M.; Samuel, T.; Margiani, P. F.; Filipe, P.; Eder, J. L.; Diego, A.; Gelson, P.; Raquel, G. J. Tetrahedron Lett. 2012, 53, 5402-5406.

https://doi.org/10.1016/j.tetlet.2012.07.118

4. Syahrul, I.; Muhammad, T.; Nor Hadiani, I. Curr Med Chem. 2015, 22, 4412-4433. https://doi.org/10.2174/0929867322666151006093930

5. Anjali, T; Meenakshi, J. Phosphorus, Sulfur, and Silicon, 2009, 184, 2835-2845. https://doi.org/10.1080/10426500802590178

6. Kalla, Redhi Mohan, N.; Shaik, I. K.; Syed, R.; Sharida, F.; Palanisamy, A.; Ola, L.; Faridah, A. Int. J. Mol. Sci. 2013, 14, 1843-1853. 
7. Deepak, K. S.; Anil, K. T.; Rashmi, S.; Reena, C.; Reyaz Ur, R.; Altaf, H.; Baldev, S.; Anindya, G.; Inshad, A. K.; Debaraj, M. Med Chem Res. 2014, 23(4), 1643-1653.

https://doi.org/10.1007/s00044-013-0764-4

8. Shiri, M.; Zolfigol, M. A.; Kruger, H. G.; Tanbakouchian, Z. Chem. Rev. 2010, 110, 2250-2293. https://doi.org/10.1021/cr900195a

9. Inamoto, T.; Papineni, S.; Chintharlapalli, S.; Cho, S. D.; Safe, S.; Kamat, A. M. Mol. Cancer Ther. 2008, 7, 3825-3833.

https://doi.org/10.1158/1535-7163.MCT-08-0730

10. York, M.; Abdelrahim, M.; Chintharlapalli, S.; Lucero, S. D.; Safe, S. Clin. Cancer Res. 2007, 13, 6743-6752. https://doi.org/10.1158/1078-0432.CCR-07-0967

11. Ichite, N.; Chougule, M. B.; Jackson, T.; Fulzele, S.V.; Safe, S.; Singh, M. Clin. Cancer Res. 2009, 15, 543-552. https://doi.org/10.1158/1078-0432.CCR-08-1558

12. Cho, S.D.; Yoon, K.; Chintharlapalli, S.; Abdelrahim, M.; Lei, P.; Hamilton, S.; Khan, S.; Ramaiah, S. K.; Safe, S. Cancer Res. 2007, 67, 674. https://doi.org/10.1158/0008-5472.CAN-06-2907

13. Imran, S.; Taha, M.; Ismail, N. H.; Fayyaz, S.; Khan, K. M.; Choudhary, M. I. Bioorg Chem 2016, 68, 90-104. https://doi.org/10.1016/j.bioorg.2016.07.011

14. Bonnesen, C.; Eggleston, I. M.; Hayes, J. D. Cancer Res. 2001, 61, 6120-6130.

15. Praveena, P. J.; Parameswaran, P. S.; Majik, M. S. Synthesis 2015, 47(13), 1827-1837. https://doi.org/10.1055/s-0034-1380415

16. Sharma, D. K.; Tripathi, A. K.; Sharma, R.; Chib, R.; Ur Rasool, R.; Hussain, A.; Singh, B.; Goswami, A.; Khan, I. A.; Mukherjee, D. Med Chem Res 2014, 23, 1643-1653.

https://doi.org/10.1007/s00044-013-0764-4

17. Beni, A. S.; Zarandi, M.; Droudgarnezhad, H. Am J of Chem and Apl 2015, 2(1), 17-22.

18. Ergindemir, H. N.; Aker, A.; Hamitbeyli, A.; Ocal, N. Molecules 2016, 21 (6), 718-730. https://doi.org/10.3390/molecules21060718

19. Peng, D.; Sun, Q.; Zhu, X.; Lin, H.; Chen, Q.; Yu, N.; Yang, W.; Yang, G. Bioorg Med Chem 2012, 20(22), 6739-6750.

https://doi.org/10.1016/j.bmc.2012.09.016

20. Zhou, Y.; Zhang, L.; Zhou, J.; Zhou, X. PCT Int. Appl. 2014, WO 2014079155 A1 20140530. 